\title{
Transcriptomic and metabolomic analyses reveal that melatonin promotes melon root development under copper stress by inhibiting jasmonic acid biosynthesis
}

\author{
Zhicheng $\mathrm{Hu}^{1}$, Qiushi $\mathrm{Fu}^{1}$, Jing Zheng ${ }^{1}$, Aiai Zhang ${ }^{1}$ and Huaisong Wang ${ }^{1}$
}

\begin{abstract}
Melatonin has been shown to alleviate the effects of abiotic stress and to regulate plant development. Copper, a common heavy metal and soil pollutant, can suppress plant growth and development. In this work, we explored the protective effects of exogenous melatonin on lateral root formation in response to copper stress using melon seeds subjected to three germination treatments: CK1 (control), CK2 (300 $\left.\mu \mathrm{mol} / \mathrm{L} \mathrm{CuSO}{ }_{4}\right)$, and MT3 $(300 \mu \mathrm{mol} / \mathrm{L}$ melatonin + $300 \mu \mathrm{mol} / \mathrm{L} \mathrm{CuSO}_{4}$ ). Melatonin pretreatment increased the antioxidant enzyme activities and root vigor, and decreased the proline and malondialdehyde (MDA) contents in the roots of copper-stressed melon seedlings. We then used transcriptomic and metabolomic analyses to explore the mechanisms by which exogenous melatonin protects against copper stress. There were 70 significant differentially expressed genes (DEGs) (28 upregulated, 42 downregulated) and 318 significantly differentially expressed metabolites (DEMs) (168 upregulated, 150 downregulated) between the MT3 and CK2 treatments. Melatonin pretreatment altered the expression of genes related to redox and cell wall formation processes. In addition, we found that members of the AP2/ERF, BBR/BPC, GRAS, and HD-ZIP transcription factor families may have vital roles in lateral root development. Melatonin also increased the level of Glutathione (GSH), which chelates excess $\mathrm{Cu}^{2+}$. The combined transcriptomic and metabolomic analysis revealed DEGs and DEMs involved in jasmonic acid (JA) biosynthesis, including four lipoxygenase-related genes and two metabolites (linoleic acid and lecithin) related to melatonin's alleviation effect on copper toxicity. This research elucidated the molecular mechanisms of melatonin's protective effects in copper-stressed melon.
\end{abstract}

\section{Introduction}

Melatonin ( $\mathrm{N}$-acetyl-5-methoxytryptamine) is an indoleamine ubiquitous in the plant kingdom ${ }^{1}$, and a modulator of multiple developmental processes and stresses ${ }^{2,3}$. Various types of research have demonstrated that melatonin can alleviate the effects of biotic and abiotic stresses, such as fungal, salt, and heavy metal stresses ${ }^{4-6}$. For example, Yin et al. ${ }^{4}$ found that apple trees pretreated with melatonin were more resistant to apple blotch than were

Correspondence: Huaisong Wang (wanghuaisong@caas.cn)

${ }^{1}$ Institute of Vegetables and Flowers, Chinese Academy of Agricultural Sciences, 100081 Beijing, China

These authors contributed equally: Zhicheng Hu, Qiushi Fu control trees. The endogenous melatonin levels of Arabidopsis serotonin $\mathrm{N}$-acetyltransferase knockout mutants were markedly lower than those of controls, resulting in susceptibility to an avirulent pathogen ${ }^{7}$. Under saline conditions, melatonin pretreatment significantly alleviated the inhibition of Malus hupehensis growth and may have reduced oxidative damage by enhancing antioxidative enzyme activities or eliminating $\mathrm{H}_{2} \mathrm{O}_{2}{ }^{5}$. Similarly, exogenous melatonin could alleviate the toxicity of cadmium stress in Ulva sp., a green macroalga ${ }^{8}$.

Melon (Cucumis melo L.) belongs to the Cucurbitaceae family and is widely planted throughout the world. Heavy metals, especially copper, have increasingly infiltrated

\section{(c) The Author(s) 2020}

(c) (i) Open Access This article is licensed under a Creative Commons Attribution 4.0 International License, which permits use, sharing, adaptation, distribution and reproduction cc) in any medium or format, as long as you give appropriate credit to the original author(s) and the source, provide a link to the Creative Commons license, and indicate if changes were made. The images or other third party material in this article are included in the article's Creative Commons license, unless indicated otherwise in a credit line to the material. If material is not included in the article's Creative Commons license and your intended use is not permitted by statutory regulation or exceeds the permitted use, you will need to obtain permission directly from the copyright holder. To view a copy of this license, visit http://creativecommons.org/licenses/by/4.0/. 
both soil and water, becoming one of the most serious current environmental problems ${ }^{6}$. At low concentrations, copper is an essential microelement for plants ${ }^{9}$, whereas at high concentrations, it has physiological and biochemical toxicity effects on plants, as shown by increased generation of reactive oxygen species (ROS), disruption of protein structure, inactivation of enzymes, and so on ${ }^{10}$. In copper-stressed Arabidopsis thaliana, root and shoot lengths and chlorophyll content levels decreased, whereas the anthocyanin content increased ${ }^{11}$. High copper concentrations inhibited root and stem growth in Arabidopsis and reduced cell elongation, division, and expansion ${ }^{12}$. In addition, $\mathrm{Cu}^{2+}$ significantly induced protein oxidation and inhibited root growth in Panax ginseng ${ }^{13}$. Some research has suggested that the application of exogenous melatonin was able to protect plants from copper stress toxicity. Exogenous melatonin increased the tolerance of red cabbage seedlings to copper stress by decreasing lipid peroxidation, thus promoting seed germination and seedling growth ${ }^{6}$. Melatonin diminished the oxidative stress and proline accumulation caused by $\mathrm{CuSO}_{4}$, indicating that melatonin had a protective effect on canola plants under excessive copper ${ }^{14}$.

Roots not only are essential organs that absorb water and minerals from the soil, thus enabling plant growth and development, but also, importantly, are involved in the perception of stress signals ${ }^{15,16}$. Therefore, roots have a key role in the acclimation of plants to adverse conditions. Lateral roots are an important part of root systems, vastly increasing their surface area and mechanical strength ${ }^{17}$. Some studies have reported inhibitory effects of excessive copper on root development in different plant species, including Arabidopsis ${ }^{12}$, Panax ginseng ${ }^{13}$, radish $^{18}$, citrus ${ }^{19}$, and sorghum ${ }^{20}$. The application of exogenous melatonin can promote root development, especially lateral root development. Melatoninoverexpressing transgenic rice seedlings showed more root formation and chlorophyll synthesis than did wildtype seedlings under cold stress ${ }^{21}$. In addition, application of melatonin to lupin promoted both morphogenesis of root primordia and the number of adventitious roots and lateral roots, as observed after indole-3-acetic acid (IAA) application ${ }^{22}$. Moreover, at low concentrations, exogenous melatonin promoted root growth of in vitro shoot tip explants of sweet cherry rootstocks, again with an effect similar to that of IAA ${ }^{23}$.

High copper levels are toxic to plants. Copper has increasingly infiltrated soil and water, seriously affecting plant growth and development ${ }^{6,10}$. Melatonin can increase plant tolerance to abiotic stress by decreasing oxidative damage and promoting root development. However, few studies have focused on the protective effects of exogenous melatonin on melon root formation under copper stress. In addition, the exact protective mechanisms elicited by exogenous melatonin remain unclear. In this work, we measured the root morphology and physiological traits, including antioxidant enzyme activities, MDA content, proline content, and root vigor, of plants subjected to eight treatments, and we ultimately selected seeds pretreated with $100 \mu \mathrm{mol} / \mathrm{L}$ melatonin from among six melatonin treatments to explore the mechanism by which melatonin protects melon roots from copper stress. We then combined transcriptomic and metabolomic analyses to compare the gene expression and metabolomic profiles between melatonin pretreatment and nonpretreatment groups under copper stress. The aim of this work was to identify the mechanisms of the protective effects of exogenous melatonin on the roots of melon seedlings under copper stress, which may be useful for the development of effective control measures against copper stress.

\section{Results \\ Melatonin promoted melon root development under copper stress}

The root morphology of melon seedlings under different treatments showed obvious differences. Untreated control (CK1) seedlings had long primary roots and many lateral roots. In comparison, the primary roots of copperstressed (CK2) seedlings were shorter, with fewer lateral roots (Fig. 1). With respect to the CK2 seedlings, their root length, root surface area, and number of root tips decreased by $62 \%, 52 \%$, and $70 \%$, respectively, whereas the root average diameter compared with that of the CK1 seedlings increased by $40 \%$ (Table 1). Melatonin pretreatment promoted the development of melon roots (Fig. 1). In the melatonin pretreatment groups, the root surface area and root length increased significantly, especially between seedlings pretreated with $100 \mu \mathrm{mol} / \mathrm{L}$ melatonin (MT3) compared with the CK2 seedlings. The root surface area and root length of the MT3 seedlings increased by $183 \%$ and $168 \%$, respectively. Excluding $10 \mu \mathrm{mol} / \mathrm{L}$ melatonin (MT1) seedlings, compared with that of the CK2 seedlings, the root volume of melatoninpretreated seedlings significantly increased by $200 \%$, $250 \%, 150 \%, 150 \%$, and $150 \%$ under pretreatment with 50 , $100,300,500$, and $800 \mu \mathrm{mol} / \mathrm{L}$ melatonin, respectively, corresponding to the MT2-MT6 treatments. The average root diameter of the MT1-MT6 seedlings increased by $10 \%, 6 \%, 18 \%, 6 \%, 16 \%$, and $14 \%$, respectively, relative to that of the CK2 seedlings. The number of root tips number of the MT2-MT6 seedlings increased by $62 \%$, $54 \%, 70 \%, 28 \%$, and $13 \%$, respectively, whereas that of the MT1 seedlings decreased by $5 \%$, compared with that of the CK2 seedlings (Table 1). The MT1-MT6 seedlings revealed that melatonin pretreatment, especially the MT3 treatment, significantly promoted root development under $\mathrm{CuSO}_{4}$ stress. 

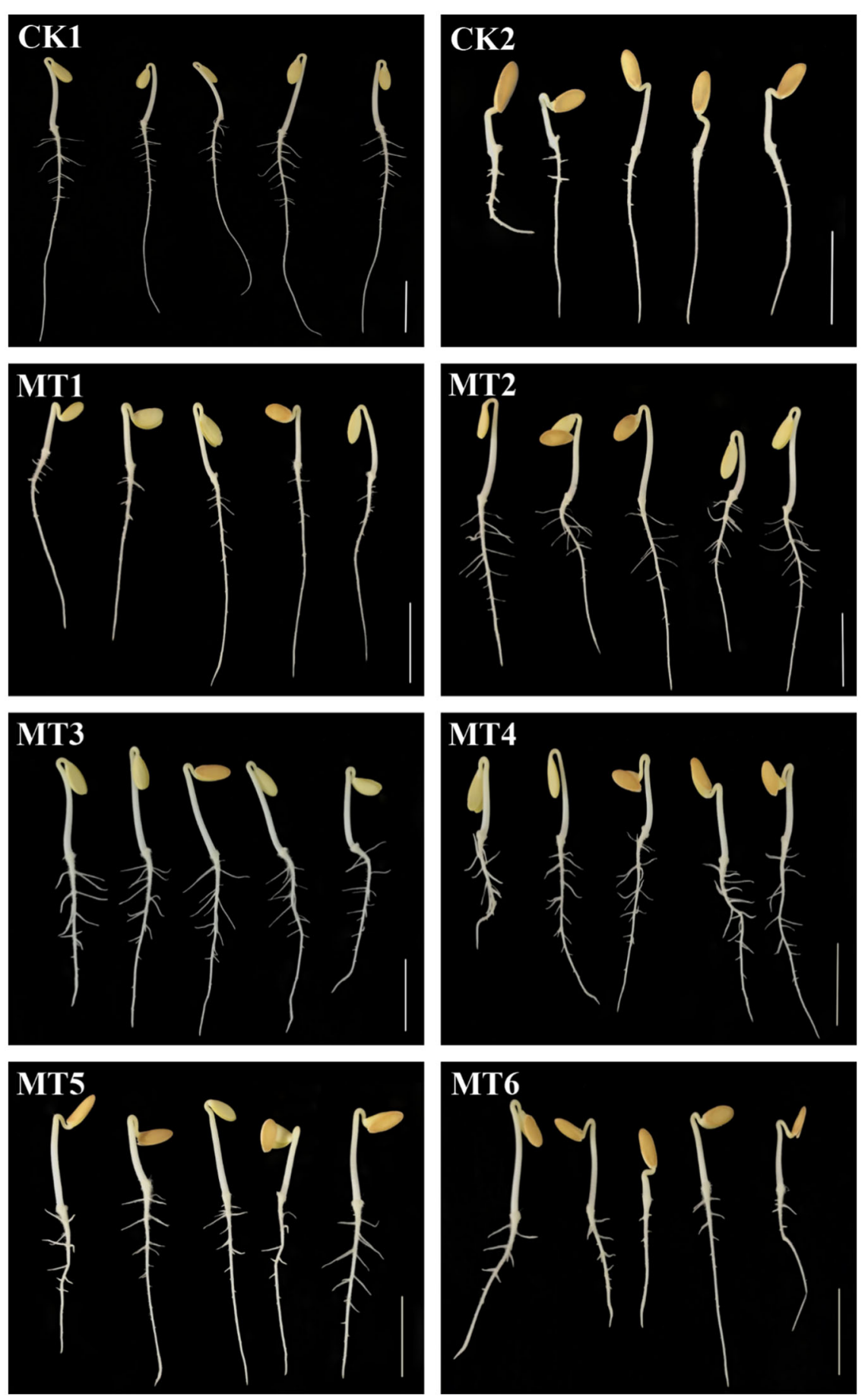

Fig. 1 Effects of melatonin on melon root development under $\mathrm{CuSO}_{4}$ stress. $\mathrm{CK} 1$ non-stressed seeds pretreated with water, $\mathrm{CK} 2300 \mu \mathrm{mol} / \mathrm{L}$ $\mathrm{CuSO}_{4}$-stressed seeds pretreated with water, MT1-MT6 $300 \mu \mathrm{mol} / \mathrm{L} \mathrm{CuSO}_{4}$-stressed seeds pretreated with 10, 50, 100, 300, 500 , or $800 \mu \mathrm{mol} / \mathrm{L}$ melatonin, respectively. Scale bar $=2 \mathrm{~cm}$

Melatonin improved melon root resistance to excess copper

The activities of the antioxidant enzymes catalase $(\mathrm{CAT})$, peroxidase (POD), and superoxide dismutase
(SOD) significantly differed among the treatments. Compared with that in the CK1 seedlings, CAT, POD, and SOD activities in the CK2 seedlings decreased by $35 \%, 39 \%$, and $26 \%$, respectively. Under melatonin 
Table 1 The effect of melatonin on melon root morphology under copper stress

\begin{tabular}{llllll}
\hline Treatment & Root length/cm & Root surface area/cm & Root volume/cm & Root average diameter/mm & Root tip number \\
\hline CK1 & $28.11 \pm 6.15 \mathrm{a}$ & $3.09 \pm 0.60 \mathrm{a}$ & $0.04 \pm 0.01 \mathrm{~b}$ & $0.35 \pm 0.03 \mathrm{c}$ & $77.00 \pm 13.78 \mathrm{a}$ \\
CK2 & $10.68 \pm 1.49 \mathrm{~b}$ & $1.49 \pm 0.19 \mathrm{c}$ & $0.02 \pm 0.00 \mathrm{~b}$ & $0.49 \pm 0.06 \mathrm{~b}$ & $23.40 \pm 7.70 \mathrm{C}$ \\
MT1 & $15.00 \pm 3.90 \mathrm{~b}$ & $2.35 \pm 0.65 \mathrm{~b}$ & $0.04 \pm 0.01 \mathrm{~b}$ & $0.54 \pm 0.02 \mathrm{a}$ & $22.20 \pm 2.68 \mathrm{c}$ \\
MT2 & $27.43 \pm 11.84 \mathrm{a}$ & $3.82 \pm 1.80 \mathrm{a}$ & $0.06 \pm 0.04 \mathrm{a}$ & $0.52 \pm 0.82 \mathrm{a}$ & $38.00 \pm 5.48 \mathrm{~b}$ \\
MT3 & $28.67 \pm 9.15 \mathrm{a}$ & $4.21 \pm 1.15 \mathrm{a}$ & $0.07 \pm 0.03 \mathrm{a}$ & $0.58 \pm 0.08 \mathrm{a}$ & $36.00 \pm 7.18 \mathrm{~b}$ \\
MT4 & $27.41 \pm 10.18 \mathrm{a}$ & $3.57 \pm 1.20 \mathrm{a}$ & $0.05 \pm 0.02 \mathrm{a}$ & $0.52 \pm 0.07 \mathrm{a}$ & $39.80 \pm 8.50 \mathrm{~b}$ \\
MT5 & $15.89 \pm 5.57 \mathrm{~b}$ & $2.73 \pm 0.36 \mathrm{~b}$ & $0.05 \pm 0.01 \mathrm{a}$ & $0.57 \pm 0.06 \mathrm{a}$ & $30.00 \pm 7.48 \mathrm{bc}$ \\
MT6 & $17.52 \pm 3.94 \mathrm{~b}$ & $2.84 \pm 0.27 \mathrm{~b}$ & $0.05 \pm 0.01 \mathrm{a}$ & $0.56 \pm 0.08 \mathrm{a}$ & $26.40 \pm 8.44 \mathrm{c}$ \\
\hline
\end{tabular}

Different letters within the same column indicate significant differences ( $p<0.05$, least significant difference test).

CK1 non-stressed seeds pretreated with water, CK2 $300 \mu \mathrm{mol} / \mathrm{L} \mathrm{CuSO}{ }_{4}$-stressed seeds pretreated with water, MT1-MT6 $300 \mu \mathrm{mol} / \mathrm{L}$ CuSO ${ }_{4}$-stressed seeds pretreated with $10,50,100,300,500$, or $800 \mu \mathrm{mol} / \mathrm{L}$ melatonin, respectively.

pretreatment, the activities of antioxidant enzymes were elevated compared with those in the CK2 seedlings: the CAT activity of the MT1-MT6 seedlings increased by $13.82 \%, 50.88 \%, 49.12 \%, 32.65 \%, 25.29 \%$, and $23.24 \%$, respectively, the POD activity of the MT1-MT6 seedlings increased by $16.17 \%, 30.25 \%, 66.13 \%, 65.21 \%, 65.69 \%$, and $61.46 \%$, respectively, and the SOD activity of the MT1-MT6 seedlings increased by $14 \%, 26 \%, 51 \%, 41 \%$, $25 \%$, and $21 \%$, respectively (Fig. $2 \mathrm{a}-\mathrm{c}$ ).

The MDA content of the CK2 seedlings increased by $40 \%$ compared with that of the CK1 seedlings. In the MT1-MT6 seedlings, the MDA content decreased by $16.17 \%, 29.17 \%, 46.67 \%, 40.64 \%, 39.58 \%$, and $30.10 \%$, respectively, compared to that in the CK2 seedlings (Fig. 2d).

Compared with that in the CK1 seedlings, the proline content in the CK2 seedlings increased by $80 \%$. Melatonin pretreatment decreased the proline content by $32.21 \%$, $38.68 \%, 49.81 \%, 55.68 \%, 51.73 \%$, and $44.07 \%$ in the MT1-MT6 seedlings, respectively, compared with that in the CK2 seedlings (Fig. 2e).

The root vigor of the CK2 seedlings was reduced by $69 \%$ relative to that of the CK1 seedlings and increased by $61.08 \%, 86.10 \%, 140.13 \%, 119.79 \%, 118.87 \%$, and $105.29 \%$ in the MT1-MT6 seedlings, respectively, relative to that in the CK2 seedlings (Fig. 2f).

These results suggest that melatonin had a positive effect on melon root development under copper stress, with $100 \mu \mathrm{mol} / \mathrm{L}$ melatonin pretreatment displaying optimal effects.

Melatonin partly alleviated copper stress, with the best response detected for the $100 \mu \mathrm{mol} / \mathrm{L}$ (MT3) melatonin pretreatment. Therefore, we selected the CK1, CK2, and MT3 treatments for transcriptomic and metabolomic analyses to identify candidate genes and metabolites involved in the alleviation of copper toxicity in melon by melatonin.
DEGs and DEMs induced by melatonin Analysis of DEGs under melatonin pretreatment in response to copper stress

Three cDNA libraries were obtained by sequencing RNA extracted from the roots of CK1, CK2, and MT3 seedlings. Each library consisted of more than 6.3 $\mathrm{Gb}$ of clean data. More than $94.4 \%$ of the clean data had scores greater than Q30 in each library. In total, 90.64 to $92.31 \%$ of clean reads were mapped to the reference genome (Table 2).

On the basis of the sequencing of the three libraries, using a fold change $\geq 1.5$ and a false discovery rate (FDR) < 0.05 as thresholds, DEGs from each two-treatment comparison (i.e., CK1 vs. CK2, CK1 vs. MT3, CK2 vs. MT3) were obtained. On the basis of the three treatments' $\log _{10}$ (FPKM) values, a hierarchical cluster analysis of the DEGs was used to observe the overall expression pattern of the genes; green and red bands were used to represent low and high gene expression levels, respectively (Fig. 3a).

Figure $3 \mathrm{~b}$ shows the differences in DEGs among the three treatments. We detected 805 DEGs (486 genes upregulated and 319 genes downregulated relative to those in CK1) associated with copper stress between the CK1 and CK2 melon root libraries. Furthermore, a total of 70 DEGs (28 genes upregulated and 42 genes downregulated relative to those in the $\mathrm{CK} 2$ ) were detected between the CK2 and MT3 libraries, which were thus associated with the response to exogenous melatonin and the alleviation of copper toxicity by melatonin (Supplementary Table S1). These genes that responded to exogenous melatonin were considered important candidates for further investigation.

The functions of the DEGs were classified according to Gene Ontology (GO) classifications. In total, 675 DEGs between CK1 and CK2 had GO annotations, most of which were enriched in the cell part, catalytic activity, 
(a)

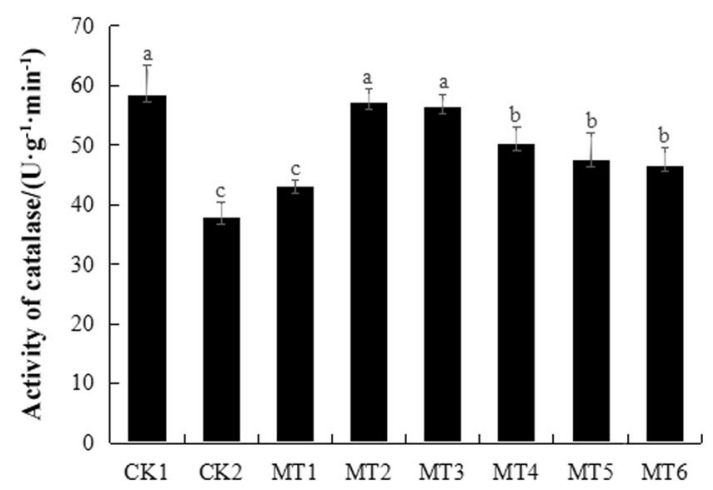

(c)

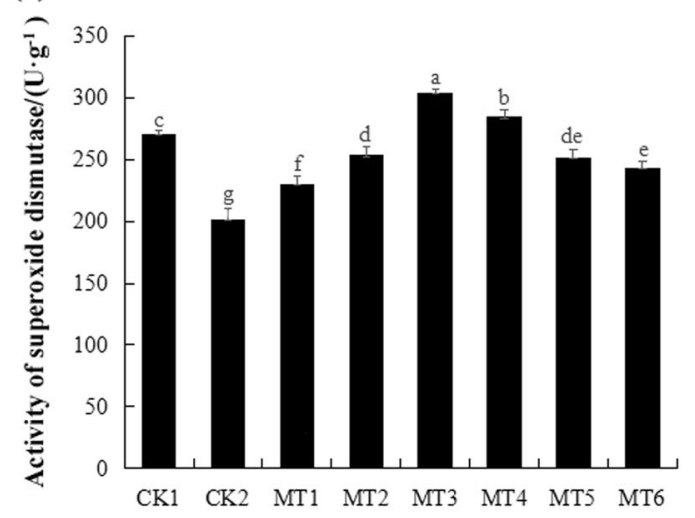

(e)

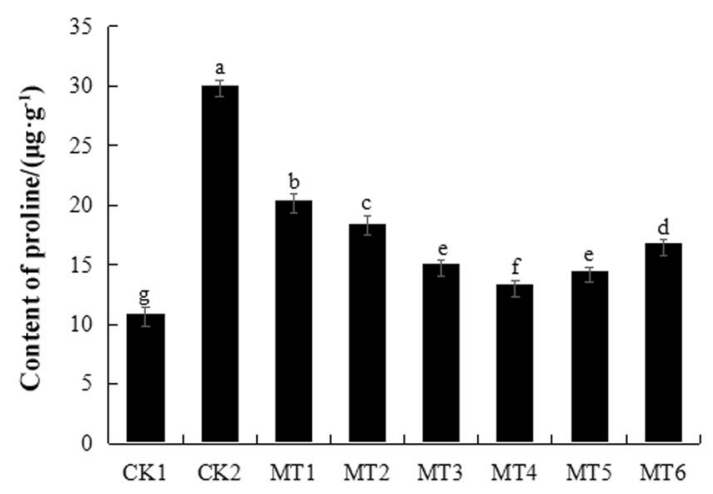

(b)

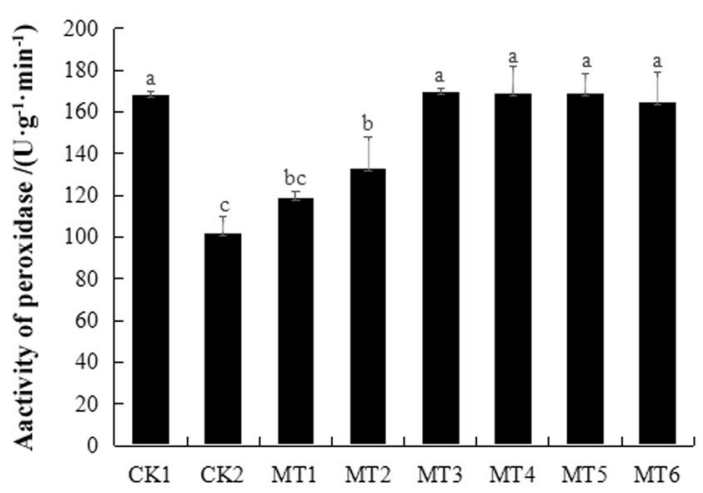

(d)

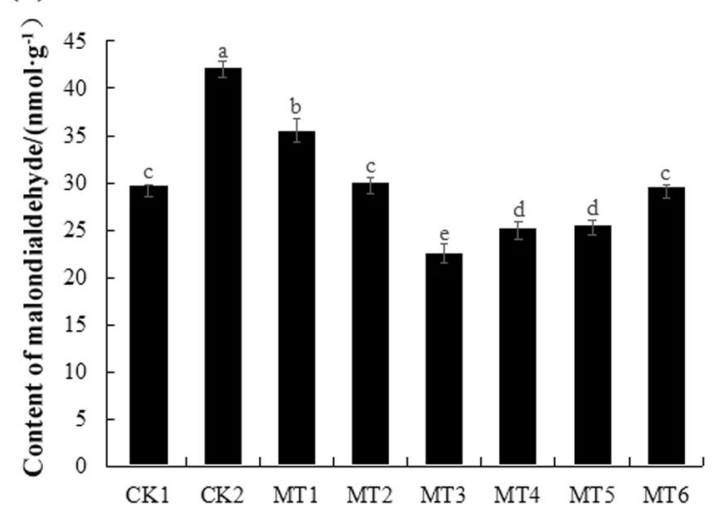

(f)

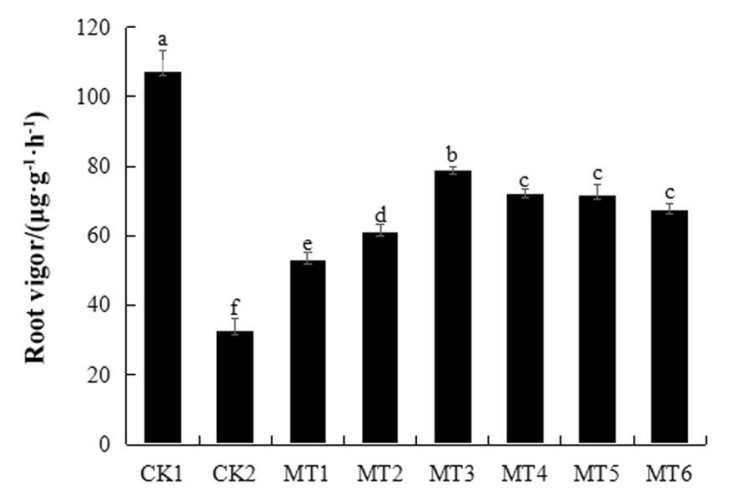

Fig. 2 Effects of melatonin on physiologic indicators in $\mathrm{CuSO}_{4}$-stressed melon. a Effect of melatonin on the activity of catalase (CAT) in melon. b Effect of melatonin on the activity of peroxidase (POD) in melon. $\mathbf{c}$ Effect of melatonin on the activity of superoxide dismutase (SOD) in melon. $\mathbf{d}$ Effect of melatonin on the content of malondialdehyde (MDA) in melon. e Effect of melatonin on the content of proline in melon. $\mathbf{f}$ Effect of melatonin on the root vigor in melon. CK1 non-stressed seeds pretreated with water, CK2 $300 \mu \mathrm{mol} / \mathrm{L} \mathrm{CuSO}_{4}$-stressed seeds pretreated with water MT1-MT6 300 mol/L CuSO ${ }_{4}$-stressed seeds pretreated with 10, 50, 100, 300, 500, or $800 \mu \mathrm{mol} / \mathrm{L}$ melatonin, respectively

single-organism process, organelle, and other functional categories. Between CK2 and MT3, there were 52 GOannotated DEGs, which were mainly categorized into the cell part, binding, single-organism process, metabolic process, and other functional categories (Fig. 3c; Supplementary Table S1).

We also conducted a Kyoto Encyclopedia of Genes and Genomes (KEGG) enrichment analysis of the DEGs to 
Table 2 Summary of sequence data

\begin{tabular}{lllllll}
\hline Sample name & Clean reads & Clean bases & Mapped reads & Error rate (\%) & Q30 (\%) & GC content (\%) \\
\hline CK1 & $21,193,701$ & $6,349,936,614$ & $41,738,693(92.31 \%)$ & 0.1 & 94.47 & 42.48 \\
CK2 & $22,601,871$ & $6,757,021,369$ & $38,414,231(90.64 \%)$ & 0.1 & 95.23 & 42.94 \\
MT3 & $22,117,437$ & $6,621,011,786$ & $40,581,838(91.73 \%)$ & 0.1 & 94.74 & 42.76 \\
\hline
\end{tabular}

CK1 non-stressed seeds pretreated with water, CK2 $300 \mu \mathrm{mol} / \mathrm{L} \mathrm{CuSO}_{4}$-stressed seeds pretreated with water, MT3 $300 \mu \mathrm{mol} / \mathrm{L}$ CuSO${ }_{4}$-stressed seeds pretreated with $100 \mu \mathrm{mol} / \mathrm{L}$ melatonin.

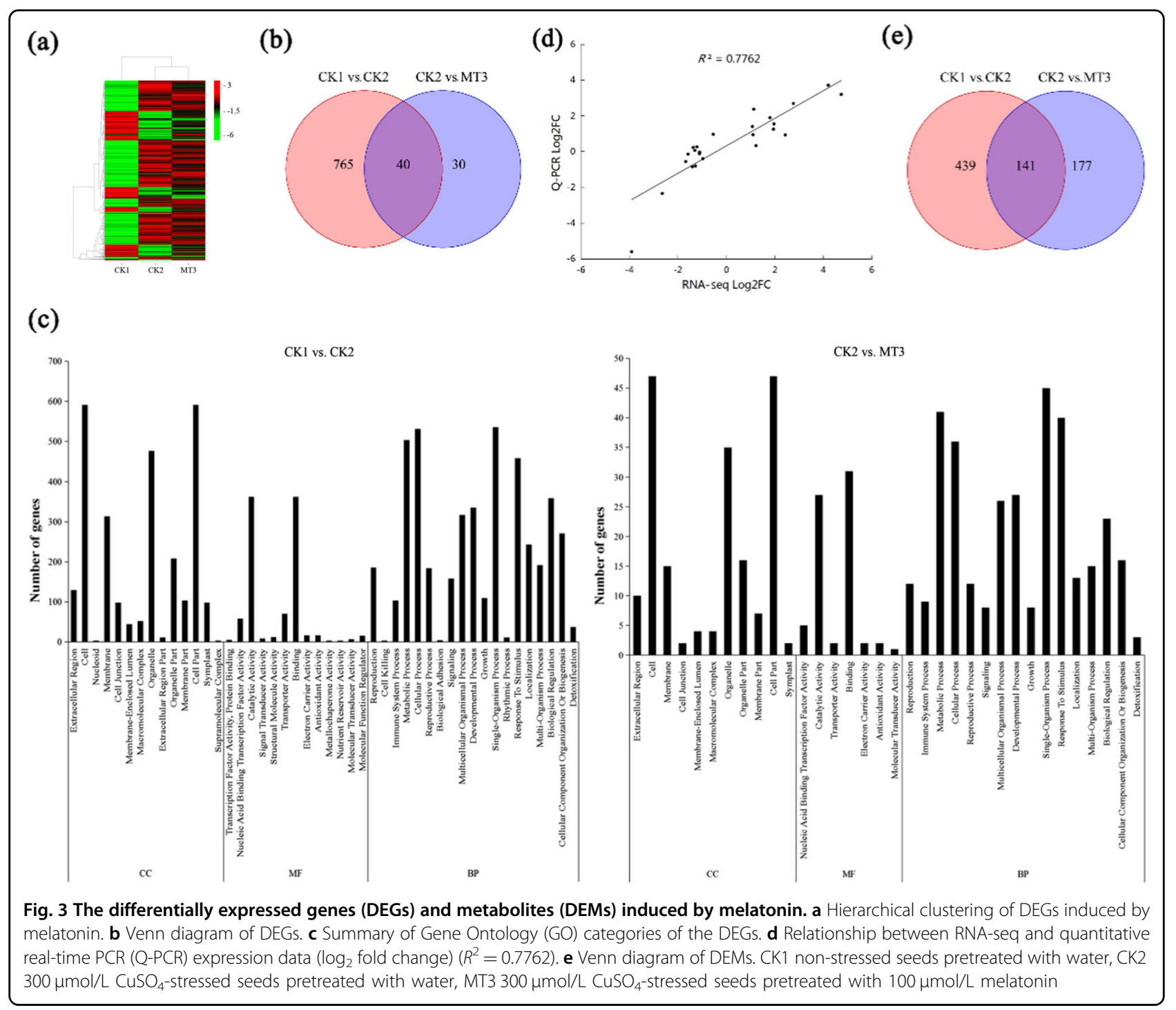

identify the main pathways active in the melatonininduced melon lateral root development process under copper stress. Between the CK1 and CK2 libraries, 159 DEGs were assigned to 78 KEGG pathways; phenylpropanoid biosynthesis (ko00940), with 28 genes (23 genes upregulated and 5 genes downregulated in CK2), and starch and sucrose metabolism (ko00500), with 17 genes (14 genes upregulated and 3 genes downregulated in CK2), were the major enriched pathways among the DEGs. Between CK2 and MT3, 11 DEGs were assigned to eight KEGG pathways; linoleic acid metabolism (ko00591), with four genes downregulated in the MT3, and endocytosis (ko04144), with three genes upregulated in the MT3, were the pathways most represented among these DEGs (Supplementary Tables S1 and S2). 
To confirm the RNA-seq data, we assessed the expression of 12 randomly selected genes using quantitative real-time PCR (Q-PCR). As shown in Fig. 3d, the Q-PCR data were consistent with the RNA-seq data, and a significant positive correlation $\left(R^{2}=0.7762\right)$ supports the reliability of the RNA-seq data.

\section{DEM analysis of melatonin pretreatment in response to copper stress}

We conducted a metabolite profiling analysis of melon root samples (from CK1, CK2, and MT3 seedlings) to assess the overall metabolic effect of melatonin pretreatment on the roots of melon seedlings under copper stress. A total of 580 metabolites that significantly differed between the CK1 and CK2 treatments were detected, with 288 upregulated and 292 downregulated relative to those in the CK1 treatment. The levels of hydroxyhydroquinone, loquatoside, lucuminamide, and galactosylceramide, among others, increased by at least twofold, whereas the levels of 4-(beta-D-ribofuranosyl) aniline $5^{\prime}$-phosphate, 4'-hydroxytrazodone, and uridine $2^{\prime}, 3^{\prime}$-cyclic phosphate, among others, decreased by more than $60 \%$ under copper stress. Between the CK2 and MT3 treatments, 318 DEMs were detected, with 168 upregulated and 150 downregulated relative to those in the CK2 treatment. Val-Phe, l-mannomethylose and dolichyl diphosphate exhibited relatively high levels under melatonin pretreatment, whereas uridine, raffinose, and hexadecanedioic acid exhibited relatively low levels (Fig. 3e; Supplementary Table S3).

To identify the main pathways involved in the roots of melon seedlings under copper stress that responded to melatonin, we mapped the differentially expressed metabolites to KEGG biological pathways. As summarized in Supplementary Table S4, 52 significantly differentially expressed metabolites between the CK1 and CK2 treatments were assigned to 67 KEGG pathways, including metabolic pathway (ko01100), biosynthesis of secondary metabolites (ko01110), biosynthesis of amino acids (ko01230), and carbon metabolism (ko01200), among others. Between the CK2 and MT3 treatments, 30 significantly differentially expressed metabolites were enriched in 44 KEGG pathways, including metabolic pathway (ko01100), biosynthesis of secondary metabolites (ko01110), pyrimidine metabolism (ko00240), and linoleic acid metabolism (ko00591), among others.

\section{Melatonin regulated antioxidant enzyme activities and redox-related gene expression to protect melon roots from copper stress}

Antioxidant enzymes have the ability to protect plants against oxidative damage induced by stress. In this study, we found that the CAT, POD and SOD activities of CK2 decreased by $35 \%, 39 \%$, and $26 \%$ relative to those of CK 1 , respectively, but those of MT3 increased by $49 \%, 66 \%$, and $51 \%$ relative to those of CK2, respectively (Fig. $4 \mathrm{a}-\mathrm{C}$ ). The MDA content exhibited the opposite trend; it increased by $40 \%$ in copper-stressed roots but decreased by $47 \%$ in melatonin-pretreated roots under copper stress (Fig. 4d). These results indicated that melatonin can alleviate the oxidative stress caused by excess copper in melon roots.

Many redox-related genes were detected, including peroxidase, lipoxygenase and glutamate dehydrogenase. Between CK1 and CK2, 51 genes were upregulated relative to those in CK1, and 26 genes were downregulated; however, between CK2 and MT3, 2 genes were upregulated relative to those in $\mathrm{CK} 2$, and 10 genes were downregulated (Supplementary Table S1). In total, nine genes that were assigned to the oxidation-reduction process (GO:0055114) were differentially expressed in all three treatments. Eight of these genes were upregulated by copper stress but downregulated by melatonin under copper stress, whereas one was downregulated by excess copper but upregulated by melatonin in $\mathrm{Cu}^{2+}$-stressed melon roots (Fig. 4e). Four lipoxygenases that were downregulated by melatonin under copper stress were also assigned to the membrane disassembly (GO:0030397) and jasmonic acid biosynthetic process (GO:0009695) functional groups (Supplementary Table S1).

\section{Melatonin altered cell wall-related gene expression to promote melon root formation}

Root development is closely related to cell wall formation. From the transcriptome analysis, we found that melatonin regulated the expression of cell wall-related genes. Between CK1 and CK2, there were 54 DEGs (13 genes upregulated and 41 genes downregulated relative to those in CK1), whereas between CK2 and MT3, there were 6 DEGs (2 genes upregulated and 4 genes downregulated relative to those in CK2). Melatonin pretreatment upregulated the expression of peroxidase and heat shock $70 \mathrm{kDa}$ protein but downregulated the expression of xyloglucan endotransglucosylase/hydrolase (XTH), dirigent protein, thaumatin-like protein, and subtilisin-like protease family protein. The expression of genes encoding dirigent protein, thaumatin-like protein and subtilisin-like protease family protein showed the opposite trend in CK2 (Supplementary Table S1; Fig. 5). These findings suggested that melatonin influenced melon root development by regulating cell wall gene expression.

\section{Melatonin regulated the expression of transcription factor genes to influence root development}

Transcription factors have a vital role in the regulation of genes in plants. We found 82 differentially expressed transcription factor genes under the copper treatment compared with the control treatment, including MYBs, 

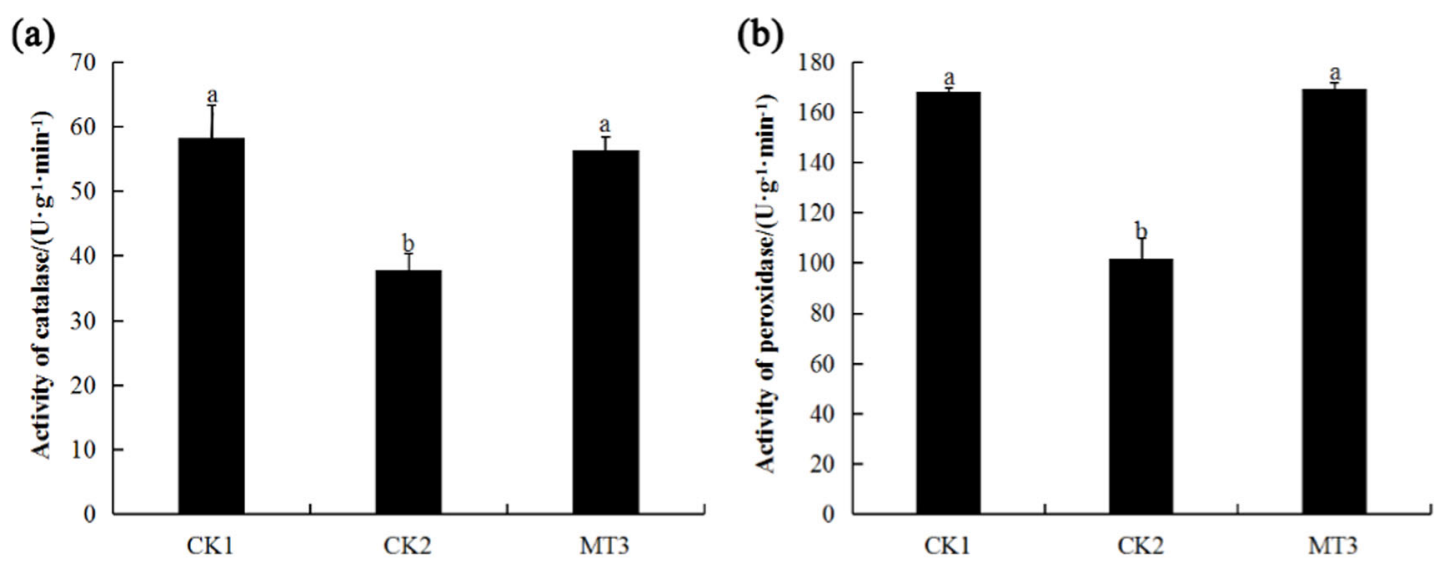

(c)

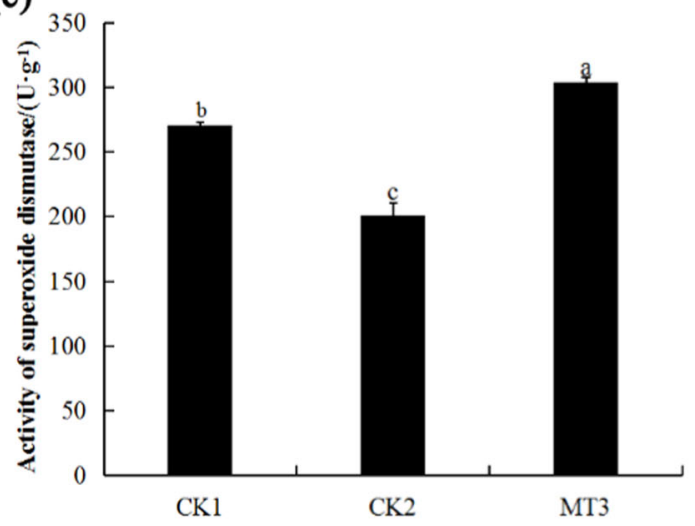

(d)

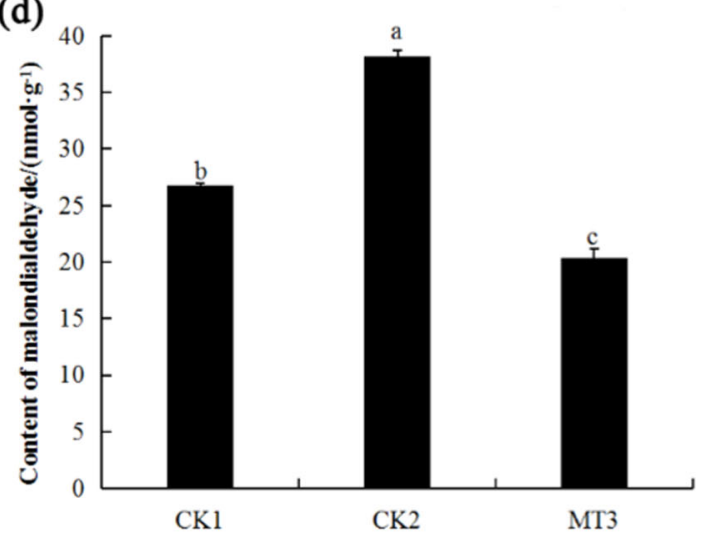

(e)

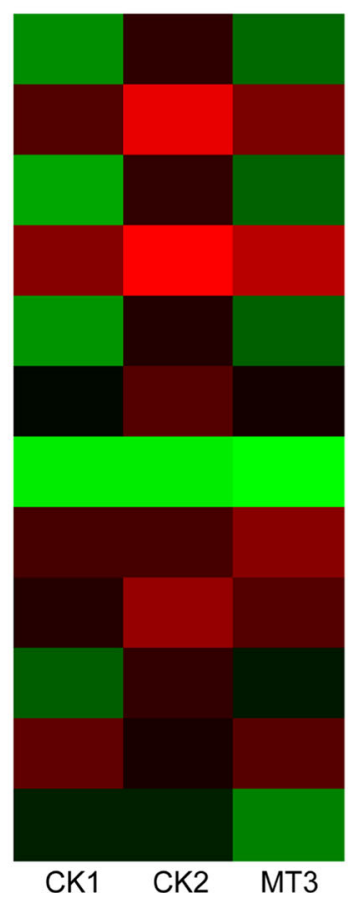

$\begin{array}{ll}\text { MELO3C000232 } & 2.5 \\ \text { MELO3C000268 } & 2.0 \\ \text { MELO3C001058 } & 1.5 \\ \text { MELO3C004245 } & 1.0 \\ \text { MELO3C004246 } & 0.5 \\ \text { MELO3C007055 } & 0.0 \\ \text { MELO3C014642 } & \\ \text { MELO3C021259 } \\ \text { MELO3C023032 } \\ \text { MELO3C024176 } \\ \text { MELO3C024896 } \\ \text { MELO3C025408 }\end{array}$

Fig. 4 Effects of melatonin on redox enzymes and genes in melon. a Effect of melatonin on CAT activity. $\mathbf{b}$ Effect of melatonin on POD activity. c Effect of melatonin on SOD activity. $\mathbf{d}$ Effect of melatonin on MDA content in melon. e Effects of melatonin on the expression of redox genes in

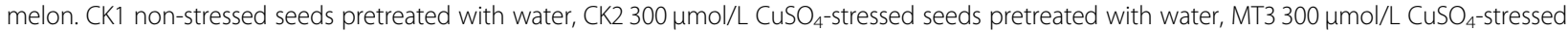
seeds pretreated with $100 \mu \mathrm{mol} / \mathrm{L}$ melatonin 


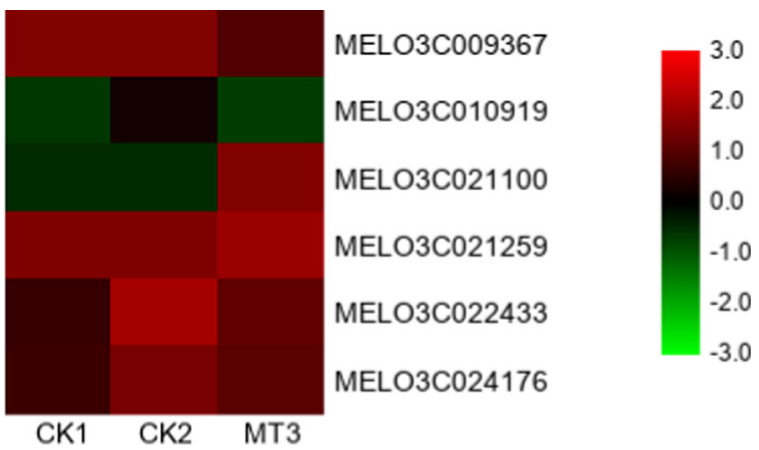

Fig. 5 Effects of melatonin on the expression of cell wall-related genes in melon. CK1 non-stressed seeds pretreated with water, CK2 $300 \mu$ mol/L $\mathrm{CuSO}_{4}$-stressed seeds pretreated with water, MT3 $300 \mu \mathrm{mol} / \mathrm{L} \mathrm{CuSO}{ }_{4}$-stressed seeds pretreated with $100 \mu \mathrm{mol} / \mathrm{L}$ melatonin

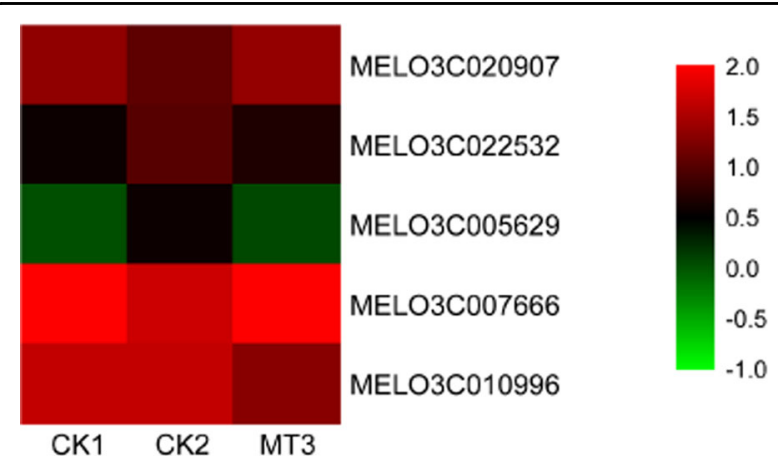

Fig. 6 Effects of melatonin on the expression of transcription factor genes in melon. CK1 non-stressed seeds pretreated with water, CK2 $300 \mu \mathrm{mol} / \mathrm{L} \mathrm{CuSO}_{4}$-stressed seeds pretreated with water, MT3 $300 \mu \mathrm{mol} / \mathrm{L} \mathrm{CuSO}_{4}$-stressed seeds pretreated with $100 \mu \mathrm{mol} / \mathrm{L}$ melatonin

NACs, WRKYs, and AP2/ERFs. In addition, five transcription factor genes were differentially expressed between melatonin-pretreated and non-pretreated roots under copper stress, including $A P 2 / E R F \mathrm{~s}, B B R / B P C \mathrm{~s}$, GRASs, and HD-ZIPs (Supplementary Table S5). In the melatonin-pretreated samples, $A P 2 / E R F \mathrm{~s}$ and $B B R / B P C \mathrm{~s}$ were significantly downregulated, whereas GRASs were markedly upregulated. We found two significantly differentially expressed $H D-Z I P \mathrm{~s}$; one was upregulated by melatonin, whereas the other was downregulated. Four of these genes showed the opposite trend in CK2 (Fig. 6; Supplementary Table S5).

Melatonin increased glutathione levels to promote copper chelation

GSH can chelate $\mathrm{Cu}^{2+}$ in cucumber under copper stress $^{24}$. Our results showed that GSH exhibited different levels in different treatments. Compared with that in the CK1, the level of glutathione disulfide in the CK2 increased by a factor of 1.06 . Compared with that in the CK2, the level of GSH in the MT3 increased by $28 \%$. In this study, pretreatment with or without melatonin increased GSH levels in the roots of melon seedlings under copper stress (Supplementary Table S3).

\section{Melatonin regulated genes and metabolites involved in JA biosynthesis to promote melon root development}

JA can inhibit plant roots by inhibiting root cell elongation, cell division ${ }^{25}$, and ROS damage ${ }^{26}$. Lipoxygenase (LOX) is the key enzyme involved in JA biosynthesis ${ }^{27,28}$. The major substrates for LOX are linoleic acid and linolenic acid ${ }^{29}$. A combined transcriptomic and metabolic analysis can better explain the transcriptional regulation of metabolic pathways. We simultaneously mapped differentially expressed genes and metabolites from the same treatment groups to KEGG pathways to clarify the relationships between genes and metabolites. Between CK2 and MT3, differentially expressed genes and metabolites were assigned only to linoleic acid metabolism (ko00591), which included four lipoxygenase-related genes (MELO3C000232, MELO3C000268, MELO3C001058, and MELO3C004245) and two metabolites (linoleic acid and lecithin), indicating that melatonin may have alleviated the effects of copper stress on roots by stimulating the lipoxygenase pathway (Supplementary Table S6). The four LOX-related genes were also enriched for linoleate the 13S-lipoxygenase activity (GO:0016165), linoleate 9Slipoxygenase activity (GO:1990136), jasmonic acid biosynthetic process (GO:0009695), and membrane disassembly (GO:0030397) functional groups (Supplementary Table S1).

In the linoleic acid metabolism pathway, between CK1 and CK2, seven genes (MELO3C000268, MELO3C001058, MELO3C004245, MELO3C014629, MELO3C000232, MELO3C014632, and Cucumis_melo_newGene_1656) and linoleic acid levels were upregulated relative to those of CK1, whereas lecithin levels were downregulated 

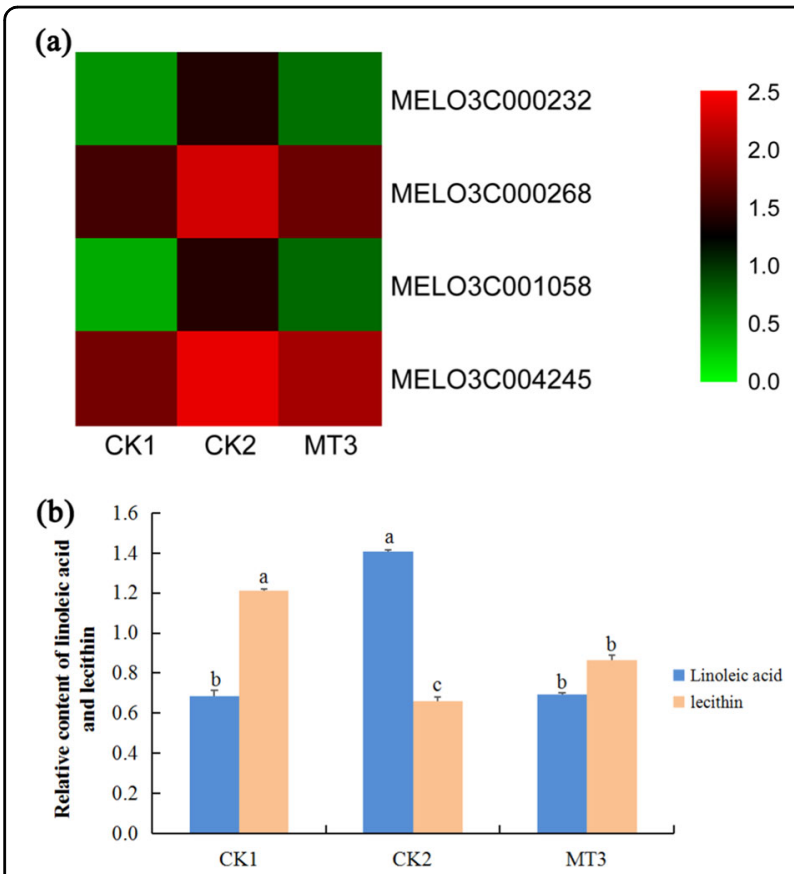

Fig. 7 Effects of melatonin on genes and metabolites involved in JA biosynthesis in melon. a Hierarchical clustering of DEGs invovled in JA biosynthesis in melon. $\mathbf{b}$ The relative contents of linoleic acid and lecithin in melon. The relative concentration values increased 1000-fold in each treatment. CK1 non-stressed seeds pretreated with water, $\mathrm{CK} 2300 \mu \mathrm{mol} / \mathrm{L} \mathrm{CuSO}_{4}$-stressed seeds pretreated with water, MT3 $300 \mu \mathrm{mol} / \mathrm{L} \mathrm{CuSO}_{4}$-stressed seeds pretreated with $100 \mu \mathrm{mol} / \mathrm{L}$ melatonin

(Supplementary Tables S1, S3, and S4). Compared with that in CK1, the expression of MELO3C000268, MELO3C001058, MELO3C004245, MELO3C014629, MELO3C000232, MELO3C014632, and Cucumis_melo_newGene_1656 in CK2 increased by factors of 4.16, 15.42, $2.72,1.91,9.60,2.41$, and 8.32 , respectively; at the same time, linoleic acid levels increased by $106 \%$, whereas lecithin levels decreased by $45 \%$. Between CK2 and MT3, four genes (MELO3C000268, MELO3C001058, MELO3C004245, and MELO3CO00232) and linoleic acid were downregulated relative to those in CK2, whereas lecithin was upregulated (Fig. 7; Supplementary Tables S1, S3, and S4). Compared with that in the CK2, the expression of MELO3COOO268, MELO3C001058, MELO3C004245, and MELO3C000232 in the MT3 decreased by $69 \%, 83 \%, 53 \%$, and $84 \%$, respectively; at the same time, linoleic acid levels decreased by $51 \%$, and lecithin levels increased by 31\% (Fig. 7; Supplementary Tables S1 and S3). These results suggested that melatonin downregulated the level of linoleic acid and the expression of lipoxygenase-related genes, thus inhibiting the biosynthesis of JA.

\section{Discussion}

As an important antioxidant and radical scavenger, melatonin can maintain the dynamic balance of free radicals and can reduce oxidative damage to plants caused by stress. Li et al. ${ }^{5}$ found that melatonin alleviated oxidative damage caused by salinity, perhaps by directly enhancing antioxidative enzyme activities or scavenging $\mathrm{H}_{2} \mathrm{O}_{2}$. Shi et al. ${ }^{30}$ found that exogenous melatonin improved the resistance of bermudagrass to drought, salt, and cold stress by enhancing antioxidative enzyme activities (i.e., POD, SOD, and CAT) and alleviating the reactive oxygen species burst. Compared with wild-type rice, melatonin-rich transgenic rice seedlings exhibited more resistance to oxidative stress induced by herbicides $^{31}$. In this study, melatonin alleviated copper stress by regulating redox reactions in melon roots.

As shown in Figs. 1 and 2, copper toxicity decreased CAT, SOD, and POD activities; increased the MDA content; and inhibited lateral root development. Overall, these results indicated that copper harmed melon root systems. Similarly, copper stress can lead to changes in lipid peroxidation, antioxidant enzyme activities, and glutathione content in Brassica juncea ${ }^{32}$. Copper also affects root meristem cell proliferation, thus impacting maize root growth, and under excess copper conditions, plasmalemmas, mitochondrial membranes, and endoplasmic reticula were disturbed ${ }^{33}$. In contrast, melatoninpretreated samples exhibited better melon lateral root development; higher activities of CAT, SOD, and POD; and a lower MDA content. These results indicated that melatonin pretreatment obviously alleviated the inhibition of melon lateral root development caused by copper stress. Thus, melatonin had a positive effect on melon root development in a concentration-dependent manner under $\mathrm{CuSO}_{4}$ stress, and the MT3 treatment best alleviated copper toxicity. This experiment demonstrated that root development of melon under $\mathrm{CuSO}_{4}$-stress was promoted by a low concentration of melatonin and inhibited by a high concentration of melatonin, as has been similarly observed with auxin, in agreement with research by Sarropoulou et al. ${ }^{23}$

We detected 12 redox-related genes with significant differential expression between the CK2 and MT3 treatments. One of them is a peroxidase-encoding gene that was upregulated in the MT3 treatment, reflecting the peroxidase activity observed in the roots. Peroxidases are present in almost all plant tissues and are particularly abundant in $\operatorname{roots}^{34}$; as important plant enzymes, peroxidases are involved in many physiological processes ${ }^{35}$. AtPrx33 and AtPrx34, two highly homologous Arabidopsis peroxidase-encoding genes, are involved in cell elongation. AtPrx34-overexpressing seedlings had significantly longer roots, whereas seedlings with reduced 
AtPrx33 or AtPrx34 expression had shorter roots ${ }^{36}$. Exogenous melatonin pretreatment upregulated peroxidase-related genes and increased peroxidase activity in cucumber roots, thus promoting lateral root formation in cucumber plants under salt stress ${ }^{37}$. Four LOX genes were downregulated by melatonin in this study. LOXs catalyze the oxygenation of polyunsaturated fatty acids, including linoleic acid, and degrade lipid bilayers and cytomembranes ${ }^{38}$. In addition, superoxide anion, peroxide, and free radicals generated by LOXs are cytotoxic and capable of damaging membranes, proteins, and DNA ${ }^{39}$. Researchers have detected the upregulation of LOX transcript levels in various plant species under different forms of stress, including wounding, cold, desiccation, and salt stress ${ }^{40-43}$. Melatonin alleviated oxidative damage by increasing antioxidant enzyme activities, decreasing MDA levels and regulating redox gene expression.

In plants, LOX is involved in polyunsaturated fatty acid metabolism and is the key enzyme involved in lipid degradation and JA biosynthesis ${ }^{27,28}$. Lecithinase, with lecithin as a substrate, catalyzes the biosynthesis of linoleic acid, and LOX and other enzymes catalyze the conversion of linoleic acid to synthesize $\mathrm{JA}^{28}$. Leaves of transgenic Arabidopsis that lacked LOX2 accumulated less JA than did control plants in response to wounding ${ }^{44}$. $\mathrm{JA}$ is an endogenous growth-regulating substance in plants, with a vital role in plant growth ${ }^{45}$, and acts as a signaling molecule involved in stress responses ${ }^{46,47}$. Under stress, the accumulation of JA largely increases; for example, tobacco infected with Pseudomonas syringae pv. phaseolicola showed increased JA accumulation and 13LOX expression in leaves within 3-9 $\mathrm{h}^{48}$. In addition, studies have shown that the restraint of plant root growth is controlled by JA. JA has been shown to exert a repressive effect on Arabidopsis primary root growth ${ }^{49}$. JA inhibited rice roots by inhibiting root cell elongation, cell division $^{25}$, and ROS damage ${ }^{26}$. In our study, the combined comparative transcriptomic and metabolic analysis between CK2 and MT3 revealed that four LOX-related genes and linoleic acid levels were downregulated in MT3, whereas lecithin levels were upregulated. Melatonin pretreatment inhibited linoleic acid biosynthesis and downregulated $L O X$ expression, thus controlling the JA content in the roots of melon seedlings under copper stress. Melatonin promoted melon lateral root development under copper stress by reducing both damage to LOX and the inhibition of JA.

The expression of cell wall-related genes was closely associated with melon lateral root formation. Melatonin regulated cell wall formation, thus affecting lateral root development. Between CK2 and MT3, six cell wall-related genes were significantly differentially expressed: peroxidase, xyloglucan endotransglucosylase/hydrolase (XTH), heat shock $70 \mathrm{kDa}$ protein, dirigent protein, thaumatin-like protein and subtilisin-like protease family protein. Peroxidase and heat shock $70 \mathrm{kDa}$ protein were significantly upregulated in the MT3 treatment, whereas the others were significantly downregulated. XTHs have an important role in cell wall remodeling and expansion, with $X T H$ expression changing under stress. The transcript level of maize wusl1005 [ $g f u]$, which encodes a homolog of xyloglucan endotransglycosylase, was enhanced under flooding stress $^{50}$. In Sagittaria pygmaea, anoxia increased the expression of SpXTH1 and SpXTH4 $4^{51}$. Similarly, at $24 \mathrm{~h}$ after Arabidopsis roots were treated with aluminum, the expression level of $X T H-5$ exhibited a statistically significant increase ${ }^{52}$. According to another study, two XTH proteins affected Arabidopsis root growth; compared with the controls, Arabidopsis seedlings incubated with AtXTH14 and AtXTH26 showed shorter and fewer root hairs ${ }^{53}$. Our results showed that the transcript level of $X T H$ was obviously decreased by melatonin pretreatment. In addition, $X T H$ expression was upregulated under copper stress, and $X T H$ overexpression may inhibit melon root development. It has been shown that activity of the heat shock $70 \mathrm{kDa}$ protein can lead to a large increase in root formation ${ }^{54}$. In our study, the expression of heat shock $70 \mathrm{kDa}$ protein was decreased in CK2 but increased in MT3. Thus, melatonin pretreatment can alleviate copper stress and promote melon root development by downregulating $X T H$ expression and upregulating heat shock $70 \mathrm{kDa}$ protein expression.

Transcription factors, such as activators, inhibitors, or both, control the expression of genes and influence many aspects of plant growth and development. We found that in melatonin-pretreated samples, $A P 2 / E R F \mathrm{~s}$ and $B B R /$ $B P C$ s were significantly downregulated, whereas GRASs were markedly upregulated. We identified two significantly differentially expressed $H D-Z I P$ transcription factors: one was upregulated by melatonin, whereas the other was downregulated. $A P 2 / E R F$ s have been shown to be involved in responses to multiple abiotic stresses, including drought, salt, and heat stresses ${ }^{55}$. Rice OsDRE1F overexpression can increase tolerance to salt, drought, and low temperatures in both rice and Arabidopsis $^{56} . B B R / B P C$ s are related to root development, and accordingly, the number of lateral roots and lateral root primordia was reduced in Arabidopsis bpc1-1 bpc2 bpc4 $b p c 6$ quadruple mutants, whereas no phenotypic changes were observed in single-gene-mutants ${ }^{57}$. Our results indicated that melatonin might alleviate the toxicity of copper and promote melon root development and, accordingly, reduce the expression of $A P 2 / E R F$ and $B B R / B P C$ transcription factor genes. SCARECROW is a GRAS family member, and in Arabidopsis AtSCR mutants, roots developed aberrantly ${ }^{58}$. The SCARECROW-like gene (CSSCL1) isolated from chestnut 
has been shown to have an essential role in the initial process of adventitious root formation ${ }^{59}$. In the present research, GRAS overexpression induced by melatonin may promote the development of melon roots. GLA$B R A 2$ (GL2) belongs to the class IV HD-ZIP family, and $G L 2$ is necessary for the regulation of root hair development in A. thaliana ${ }^{60,61}$. ATHB6, an HD-ZIP gene, was significantly upregulated in A. thaliana seedlings under osmotic and water deficit stress and in response to exogenous abscisic acid (ABA) treatment ${ }^{62}$. Similar to $A T H B 6$, both $A T H B 7$ and $A T H B 12$ were upregulated under water deficit ${ }^{63}$. In cucumber, CsHDZI12 was repressed under high-salinity conditions, whereas CsHDZI4 was repressed by low temperature ${ }^{64}$. In the present study, melatonin regulated the expression of HD-ZIP-related genes to promote root growth and development. These results suggest that transcription factors have an essential role in root development induced by melatonin.

GSH exists in reduced glutathione and oxidized glutathione forms and is involved in both the sequestration of heavy metals and the detoxification of $\operatorname{ROS}^{65}$. GSH can chelate excess metals in plants. In cadmium-exposed oat roots, GSH was found to be associated with cadmium ${ }^{66}$. In cucumber, GSH chelated excess $\mathrm{Cu}^{2+}$ and formed heavy metal complexes that were transported to the vacuole $^{24}$. In our study, we found that with and without melatonin pretreatment increased the level of GSH in copper-stressed melon roots. Compared with that in CK1, the GSH level in CK2 increased, which may reflect melon's defensive reaction. Melatonin pretreatment further increased the GSH level in MT3, which allowed the melon roots to develop better than those in CK2.

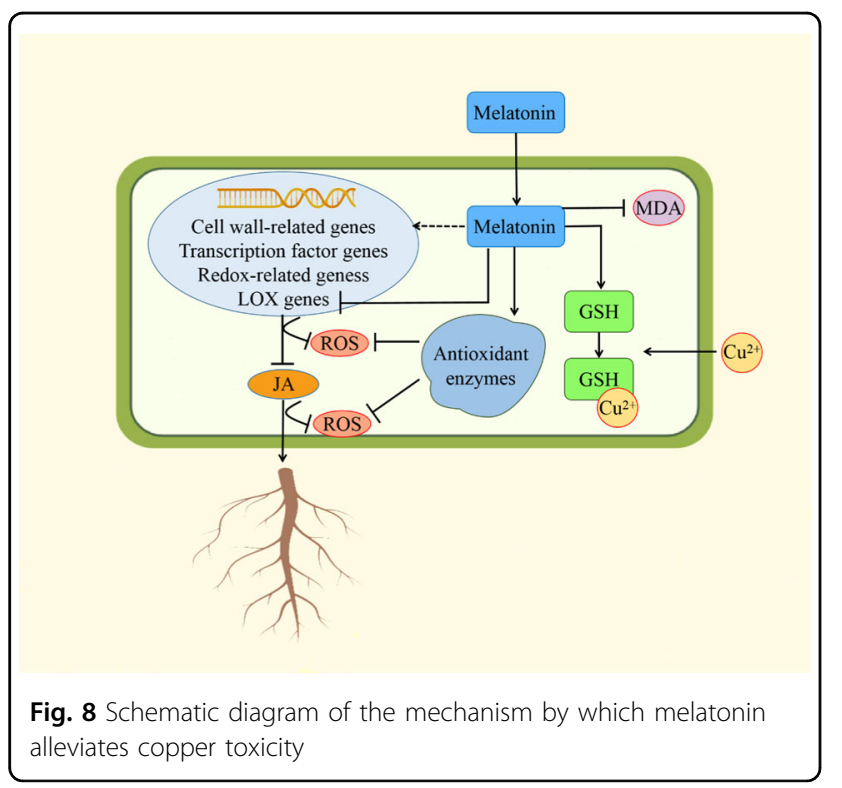

Our results suggested that melatonin could promote melon root development by regulating linoleic acid metabolism. Melatonin decreased the level of linoleic acid and the expression of four LOX-related genes, thus decreasing the JA level. Melatonin decreased ROS damage by reducing LOX-related gene expression and JA levels, regulating the expression of other redox genes and increasing antioxidant enzyme activities. Melatonin also altered the expression of genes related to cell wall formation processes and of members of the AP2/ERF, BBR/ BPC, GRAS, and HD-ZIP transcription factor families. In addition, melatonin increased the level of GSH, which was chelated excess $\mathrm{Cu}^{2+}$. In conclusion, melatonin alleviated copper toxicity and promoted melon root development via multiple mechanisms (Fig. 8).

\section{Materials and methods \\ Plant materials}

Plump melon seeds were allotted to one of eight treatments. The seeds of treatments MT1- MT6 were pretreated in melatonin solutions (concentration of 10,50 , $100,300,500$, or $800 \mu \mathrm{mol} / \mathrm{L}$, respectively) at $25^{\circ} \mathrm{C}$ for $12 \mathrm{~h}$. Seeds in the CK1 and CK2 treatments were pretreated in water at $25^{\circ} \mathrm{C}$ for $12 \mathrm{~h}$. Afterwards, the CK2 and MT1-MT6 seeds were germinated on three layers of filter paper moistened with a $300 \mu \mathrm{mol} / \mathrm{L} \mathrm{CuSO}_{4}$ solution, whereas the CK1 seeds were germinated on three layers of water-moistened filter paper. The seeds were then cultivated at $30^{\circ} \mathrm{C}$ in darkness for 3 days. Each treatment was replicated five times, with 1000 seeds each. The roots were separated from seedlings and used as materials for additional experiments and analysis.

\section{Measurement of antioxidant enzyme activities, proline content, malondialdehyde content, and root vigor}

In accordance the methods of $\mathrm{Li}$ et al. ${ }^{67}$ we measured antioxidant enzyme activities, proline content, MDA content, and root vigor. POD, CAT, and SOD activities were measured by guaiacol, spectrophotometry, and nitro blue tetrazolium methods, respectively. Proline and MDA contents were measured using the ninhydrin reaction and thiobarbituric acid colorimetric methods, respectively. Last, the 2,3,5-triphenyltetrazolium chloride (TTC) method was used to measure root vigor.

\section{RNA quantification and qualification}

RNA was extracted from melon root tissue, with three replicates per treatment. Total RNA was extracted using a Uniq-10 column TRIzol total RNA extraction kit. We measured RNA concentrations using a NanoDrop 2000 (Thermo Fisher Scientific, Waltham, MA, USA) and assessed the RNA integrity using a RNA Nano 6000 Assay Kit in conjunction with an Agilent Bioanalyzer 2100 system (Agilent Technologies, Santa Clara, CA, USA). 


\section{Library construction for sequencing}

RNA sample sequencing was conducted using $1 \mu \mathrm{g}$ of RNA per sample. We constructed sequencing libraries using a NEBNext Ultra RNA Library Prep Kit for Illumina (New England Biolabs, Ipswich, MA, USA) and added index codes to facilitate attributing sequences to samples. For each sample, mRNA was removed from total RNA using poly- $\mathrm{T}$ oligo-attached magnetic beads. Dissociation was performed using divalent cations under elevated temperatures. First-strand cDNA synthesis was performed using random hexamer primers and M-MuLV Reverse Transcriptase, and second-strand cDNA synthesis used DNA Polymerase I and RNase $\mathrm{H}$. The remaining overhangs were converted into blunt ends using polymerase or exonuclease activities. DNA fragments with polyadenylation at their $3^{\prime}$ ends were connected to NEBNext adaptors with a hairpin loop structure to prepare for hybridization. The fragments were purified using an AMPure XP system (Beckman Coulter, Beverly, MA, USA) to select cDNA fragments of $240 \mathrm{bp}$ in length. Adaptor-ligated and sizeselected cDNA was and $3 \mu \mathrm{L}$ of USER Enzyme (New England Biolabs) were then mixed together at $37^{\circ} \mathrm{C}$ for $15 \mathrm{~min}$, after which the mixture was incubated at $95^{\circ} \mathrm{C}$ for 5 min before PCR. PCR was performed with Index (X) Primer, Universal PCR primers, and Phusion High-Fidelity DNA polymerase. Last, we purified PCR products using an AMPure XP system and assessed library quality using an Agilent Bioanalyzer 2100 system.

\section{Clustering and sequencing}

The clustering of the index-coded samples was conducted using a cBot Cluster Generation System with a TruSeq PE Cluster Kit v4-cBot-HS (Illumina, San Diego, CA, USA) following the manufacturer's recommendations. The prepared libraries were sequenced on an Illumina platform, and paired-end reads were produced after cluster generation.

\section{Transcriptomic data analysis}

Clean reads were produced by removing low-quality reads as well as reads containing adaptor sequences or poly- $\mathrm{N}$ sequences from raw reads. Moreover, the sequence duplication level, GC content, and percentages of Q20 and Q30 reads were calculated from clean reads. High-quality clean reads were used for downstream analyses.

The clean reads were mapped to the reference genome sequence, and reads that were perfectly matched or contained one mismatch were further analyzed and annotated on the basis of the reference genome. We used HISAT2 tools to map the reads to the reference genome. Gene expression levels were estimated as fragments per kilobase of transcript per million fragments mapped (FPKM).

Differential expression analyses among the three treatments (CK1 vs. CK2, CK1 vs. MT3, CK2 vs. MT3, with three biological replicates per treatment) were conducted using the DESeq R package (1.10.1). DESeq uses a statistical model based on a negative binomial distribution to identify differentially expressed genes from digital gene expression data. Benjamini and Hochberg's approach was used to adjust $p$-values, and the FDR was controlled for by adjusting the $p$-value threshold. The fold change indicates the ratio of expression between the two treatments within a comparison. Genes with a fold change $\geq 1.5$ and an FDR $<0.05$ were considered to be DEGs.

$\mathrm{GO}$ is a set of annotation categories pertaining to the biological processes, molecular functions, and cellular components of various genes. GO enrichment analysis of the DEGs was performed using the GOseq R package ${ }^{68}$. The $\mathrm{KEGG}^{69}$ database is a resource that systematizes the highlevel functions of biological systems according to biological pathways (http://www.genome.jp/kegg/). KOBAS ${ }^{70}$ software was used to test the statistical enrichment of DEGs in the KEGG pathways.

\section{Quantitative real-time PCR validation}

Q-PCR was used to validate the RNA-seq data for 12 different genes. Specific primers were designed using Primer Premier 5 software (Premier Biosoft, Palo Alto, CA, USA) (Supplementary Table S7). The RNA samples were used to synthesize cDNA, and a StepOnePlus RealTime Fluorescent Quantitative PCR system (ABI, Foster City, CA, USA) was used to monitor the amount of DNA. Assays of each gene were repeated three times. Quantification was evaluated using the $2^{-(\Delta \Delta \mathrm{Ct})}$ method.

\section{Extraction and quantification of metabolites}

Metabolites were extracted from melon root tissue, with five replicates per treatment. Metabolites were analyzed using an Agilent 1290 ultra-high-performance liquid phase chromatograph. Together with Analyst TF 1.7 software, an AB5600 Triple TOF mass spectrometer in IDA mode was used to collect primary and secondary mass spectral data. Molecular ions with the strongest intensity and exceeding 100 were then chosen to acquire the corresponding secondary mass spectral data during each data acquisition cycle. The energy was $30 \mathrm{eV}$ in the electron impact mode, with 15 secondary mass spectra/ $50 \mathrm{~ms}$. The ESI ion source parameters were as follows: the atomization pressure (GS1) was $60 \mathrm{psi}$, auxiliary pressure was $60 \mathrm{psi}$, air curtain pressure was $35 \mathrm{psi}$, temperature was $6500^{\circ} \mathrm{C}$, and spray voltage was $-4000 \mathrm{~V}$ (negative ion mode) or $5000 \mathrm{~V}$ (positive ion mode).

The raw mass spectral data were converted into mzXML format using ProteoWizard Toolkit ${ }^{71}$. XCMS was then used for retention time correction, peak identification, peak extraction, peak integration, peak alignment, and so on. Afterward, we used an in-house $\mathrm{R}$ package and a custom-made secondary mass 
spectrometry database to identify peaks. The peak area data of a single peak or for all groups with a null value no more than $50 \%$ was retained after a single peak was filtered. The missing value in the original data was filled using the minimum value $1 / 2$ simulated method. The total ion current (TIC) of each sample was used for normalization.

\section{Metabolomic data analysis}

We used a combination of the $p$-values of Student's $t$-tests with the VIP value of the OPLS-DA model to filter differentially expressed metabolites, with additional screening criteria $(p<0.1, \mathrm{VIP}>0.1)$. We constructed metabolic pathways based on the information in the KEGG database.

\section{Combined metabolomic and transcriptomic analysis}

The metabolomic and transcriptomic data were converted to $\log 2$ values before analysis. We used Pearson correlation coefficients (PCCs) and corresponding $p$ values (PCCPs) to screen metabolites and related genes within the combined metabolomic and transcriptomic analysis; the screening criteria were $\mathrm{PCC}>0.80$ and $\mathrm{PCCP}<0.05$. To better understand the relationship between genes and metabolites, we mapped the differentially expressed genes and metabolites among the same treatments (CK1, CK2, and MT3) to their associated KEGG pathways.

\section{Acknowledgements}

This work was supported by the National Key R\&D Program of China (2018YFD0100705), the China Agriculture Research System of Watermelon and Melon (CARS-25), the Innovation Engineering Project of the Chinese Academy of Agricultural Sciences, and the Central Public-Interest Scientific Institution Basal Research Fund (1610102016026, IVF-BRF2018011).

\section{Author contributions}

Huaisong Wang and Qiushi Fu conceived and designed the research Zhicheng Hu and Qiushi Fu performed the experiments. Zhicheng Hu, Jing Zheng and Aiai Zhang analyzed the data. Zhicheng Hu, Qiushi Fu and Huaisong Wang wrote the manuscript.

\section{Conflict of interest}

The authors declare that they have no conflict of interest.

Supplementary Information accompanies this paper at (https://doi.org/ 10.1038/s41438-020-0293-5).

Received: 12 November 2019 Revised: 3 January 2020 Accepted: 20 March 2020

Published online: 01 June 2020

\section{References}

1. Reiter, R. J. et al. Phytomelatonin: assisting plants to survive and thrive. Molecules 20, 7396-7437 (2015).

2. Kolar, J. \& Machackova, I. Melatonin in higher plants: occurrence and possible functions. J. Pineal Res. 39, 333-341 (2005).

3. Tan, D. X. et al. Functional roles of melatonin in plants, and perspectives in nutritional and agricultural science. J. Exp. Bot. 63, 577-597 (2012).

4. Yin, L. et al. Exogenous melatonin improves Malus resistance to Marssonina apple blotch. J. Pineal Res. 54, 426-434 (2013).
5. Li, C. et al. The mitigation effects of exogenous melatonin on salinity-induced stress in Malus hupehensis. J. Pineal Res. 53, 298-306 (2012).

6. Posmyk, M. G. M., Hanna, K., Kazimierz, M. \& Janas, K. M. Presowing seed treatment with melatonin protects red cabbage seedlings against toxic copper ion concentrations. J. Pineal Res. 45, 24-31 (2008).

7. Lee, H. Y., Byeon, Y., Tan, D.-X., Reiter Russel, J. \& Back, K. Arabidopsis serotonin $\mathrm{N}$-acetyltransferase knockout mutant plants exhibit decreased melatonin and salicylic acid levels resulting in susceptibility to an avirulent pathogen. J. Pineal Res. 58, 291-299 (2015).

8. Tal, O., Haim, A., Harel, O. \& Gerchman, Y. Melatonin as an antioxidant and its semi-lunar rhythm in green macroalga Ulva sp. J. Exp. Bot. 62, 1903-1910 (2011).

9. Maksymiec, W. Effect of copper on cellular processes in higher plants. Photosynthetica 34, 321-342 (1997).

10. Yruela, I. Copper in plants: acquisition, transport and interactions. Funct. Plant Biol. 36, 409-430 (2009).

11. Baek, S.-A. et al. Effects of heavy metals on plant growths and pigment contents in Arabidopsis thaliana. Plant Pathol. J. 28, 446-452 (2012).

12. Kolbert, Z., Pető, A., Lehotai, N., Feigl, G. \& Erdei, L. Long-term copper $\left(\mathrm{Cu}^{2+}\right)$ exposure impacts on auxin, nitric oxide (NO) metabolism and morphology of Arabidopsis thaliana L. Plant Growth Regul. 68, 151-159 (2012).

13. Ali, M. B., Singh, N., Shohael, A. M., Hahn, E. J. \& Paek, K-Y. Phenolics metabolism and lignin synthesis in root suspension cultures of Panax ginseng in response to copper stress. Plant Sci. 171, 147-154 (2006).

14. Kholodova, V. P. et al. Exogenous melatonin protects canola plants from toxicity of excessive copper. Russian J. Plant Physiol. 65, 882-889 (2018).

15. Meister, R., Rajani, M. S., Ruzicka, D. \& Schachtman, D. P. Challenges of modifying root traits in crops for agriculture. Trends Plant Sci. 19, 779-788 (2014).

16. Giehl, R. F., Gruber, B. D. \& von Wiren, N. It's time to make changes: modulation of root system architecture by nutrient signals. J. Exp. Bot. 65, 769-778 (2014).

17. Nibau, C., Gibbs, D. J. \& Coates, J. C. Branching out in new directions: the control of root architecture by lateral root formation. N. Phytol. 179, 595-614 (2008).

18. Kovac, J., Lux, A. \& Vaculik, M. Formation of a subero-lignified apical deposit in root tip of radish (Raphanus sativus) as a response to copper stress. Ann. Bot. 122, 823-831 (2018).

19. Hippler, F. W. R. et al. Mechanisms of copper stress alleviation in Citrus trees after metal uptake by leaves or roots. Environ. Sci. Pollut. Res Int 25, 13134-13146 (2018).

20. Roy, S. K. et al. Proteome characterization of copper stress responses in the roots of sorghum. Biometals 30, 765-785 (2017).

21. Kang, K., Lee, K. S., Kim, Y. S. \& Back, K. Enhanced production of melatonin by ectopic overexpression of human serotonin $\mathrm{N}$-acetyltransferase plays a role in cold resistance in transgenic rice seedlings. J. Pineal Res. 49, 176-182 (2010).

22. Arnao, M. B. \& Josefa, H. R. Melatonin promotes adventitious- and lateral root regeneration in etiolated hypocotyls of Lupinus albus L. J. Pineal Res. 42, 147-152 (2007)

23. Sarropoulou, V. N., Therios, I. N. \& Dimassi-Theriou, K. N. Melatonin promotes adventitious root regeneration in in vitro shoot tip explants of the commercial sweet cherry rootstocks CAB-6P (Prunus cerasus L.), Gisela 6 ( $P$. cerasus $\times P$. canescens), and MXM 60 (P. avium $\times$ P. mahaleb). J. Pineal Res. 52, 38-46 (2012).

24. Cao, Y. Y. et al. Melatonin alleviates copper toxicity via improving copper sequestration and ROS scavenging in cucumber. Plant Cell Physiol. 60, 562-574 (2019)

25. Liu, L. et al. Salinity inhibits rice seed germination by reducing alpha-amylase activity via decreased bioactive gibberellin content. Front Plant Sci. 9, 275 (2018).

26. Soares, A. M. D. S., Souza, T. F. D., Jacinto, T. \& Machado, O. L. T. Effect of methyl jasmonate on antioxidative enzyme activities and on the contents of ROS and $\mathrm{H}_{2} \mathrm{O}_{2}$ in Ricinus communis leaves. Braz. J. Plant Physiol. 33, 151-158 (2010).

27. Baldwin, I. T., Schmelz, E. A. \& Zhang, Z.P. Effects of octadecanoid metabolites and inhibitors on induced nicotine accumulation in Nicotiana sylvestris. Chem. Ecol. 22, 61-74 (1996)

28. Feussner, I. \& Wasternack, C. The lipoxygenase pathway. Annu Rev. Plant Biol. 53, 275-297 (2002).

29. Wang, R. et al. A novel lipoxygenase gene from developing rice seeds confers dual position specificity and responds to wounding and insect attack. Plant Mol. Biol. 66, 401-414 (2008).

30. Shi, H. et al. Comparative physiological, metabolomic, and transcriptomic analyses reveal mechanisms of improved abiotic stress resistance in bermudagrass [Cynodon dactylon (L). Pers.] by exogenous melatonin. J. Exp. Bot. 66, 681-694 (2015). 
31. Park, S. et al. Melatonin-rich transgenic rice plants exhibit resistance to herbicide-induced oxidative stress. J. Pineal Res. 54, 258-263 (2013).

32. Devi, S. R. \& Prasad, M. N. V. Antioxidant capacity of Brassica juncea plants exposed to elevated levels of copper. Russian J. Plant Physiol. 52, 205-208 (2005).

33. Doncheva, S. Copper-induced alterations in structure and proliferation of maize root meristem cells. J. Plant Physiol. 153, $482-487$ (1998).

34. Dunand, C., Crevecoeur, M. \& Penel, C. Distribution of superoxide and hydrogen peroxide in Arabidopsis root and their influence on root development: possible interaction with peroxidases. N. Phytol. 174, 332-341 (2007).

35. Gapper, C. \& Dolan, L. Control of plant development by reactive oxygen species. Plant Physiol. 141, 341-345 (2006)

36. Passardi, F., Tognolli, M., De Meyer, M., Penel, C. \& Dunand, C. Two cell wall associated peroxidases from Arabidopsis influence root elongation. Planta 223, 965-974 (2006)

37. Zhang, N. et al. The RNA-seq approach to discriminate gene expression profiles in response to melatonin on cucumber lateral root formation. J. Pineal Res. 56, 39-50 (2014).

38. Vellosillo, T. et al. Oxylipins produced by the 9-lipoxygenase pathway in Arabidopsis regulate lateral root development and defense responses through a specific signaling cascade. Plant Cell 19, 831-846 (2007).

39. Melan, M. A. et al. An Arabidopsis thaliana lipoxygenase gene can be induced by pathogens, abscisic acid, and methyl jasmonate. Plant Physiol. 101, 441-450 (1993).

40. Porta, $\mathrm{H}$. et al. Analysis of lipoxygenase mRNA accumulation in the common bean (Phaseolus vulgaris L.) during development and under stress conditions. Plant Cell Physiol. 40, 850-858 (1999).

41. Yang, $X . Y$., Jiang, W. J. \& Yu, H. J. The expression profiling of the lipoxygenase (LOX) family genes during fruit development, abiotic stress and hormonal treatments in cucumber (Cucumis sativus L.). Int J. Mol. Sci. 13, 2481-2500 (2012).

42. Lim, C. W. et al. The pepper lipoxygenase CaLOX1 plays a role in osmotic, drought and high salinity stress response. Plant Cell Physiol. 56, 930-942 (2015)

43. Hou, Q.-z et al. Changes by cadmium stress in lipid peroxidation and activities of lipoxygenase and antioxidant enzymes in Arabidopsis are associated with extracellular ATP. Biologia 72, 1467-1474 (2017).

44. Bell, E., Creelman, R. A. \& Mullet, J. E. A chloroplast lipoxygenase is required for wound-induced jasmonic acid accumulation in Arabidopsis. Proc. Natl Acad. Sci. USA 92, 8675-8679 (1995).

45. Song, M. S., Kim, D. G. \& Lee, S. H. Isolation and characterization of a jasmonic acid carboxyl methyltransferase gene from hot pepper (Capsicum annuum L.). J. Plant Biol. 48, 292-297 (2005).

46. Farmer, E. E. \& Ryan, C. A. Octadecanoid precursors of jasmonic acid activate the synthesis of wound-inducible proteinase inhibitors. Plant Cell 4, 129-134 (1992).

47. Creelman, R. A., Tierney, M. L. \& Mullet, J. E. Jasmonic acid/methyl jasmonate accumulate in wounded soybean hypocotyls and modulate wound gene expression. Proc. Natl Acad. Sci. USA 89, 4938-4941 (1992).

48. Kenton, P., Mur, L. A. M., Atzorn, R., Wasternack, C. \& Draper, J. (-)-Jasmonic acid accumulation in tobacco hypersensitive response lesions. Mol. PlantMicrobe Interact. 12, 74-78 (1999).

49. Chen, Q. et al. The basic helix-loop-helix transcription factor MYC2 directly represses PLETHORA expression during jasmonate-mediated modulation of the root stem cell niche in Arabidopsis. Plant Cell 23, 3335-3352 (2011).

50. Saab, I. N. \& Sachs, M. M. A flooding-induced xyloglucan endo-transglycosylase homolog in maize is responsive to ethylene and associated with aerenchyma. Plant Physiol. 112, 385-391 (1996).

51. Ookawara, R., Satoh, S., Yoshioka, T. \& Ishizawa, K. Expression of alpha-expansin and xyloglucan endotransglucosylase/hydrolase genes associated with shoot elongation enhanced by anoxia, ethylene and carbon dioxide in arrowhead (Sagittaria pygmaea Miq.) tubers. Ann. Bot. 96, 693-702 (2005).

52. Yang, J. L. et al. Cell wall hemicellulose contributes significantly to aluminum adsorption and root growth in Arabidopsis. Plant Physiol. 155, 1885-1892 (2011).

53. Maris, A., Suslov, D., Fry, S. C., Verbelen, J. P. \& Vissenberg, K. Enzymic characterization of two recombinant xyloglucan endotransglucosylase/hydrolase (XTH) proteins of Arabidopsis and their effect on root growth and cell wall extension. J. Exp. Bot. 60, 3959-3972 (2009).

54. Kares, C., Prinsen, E., Van Onckelen, H. \& Otten, L. IAA synthesis and root induction with iaa genes under heat shock promoter control. Plant Mol. Biol. 15, 225-236 (1990).

55. Wan, L. et al. Identification of ERF genes in peanuts and functional analysis of AhERFO08 and AhERFO19 in abiotic stress response. Funct. Integr. Genomics 14 467-477 (2014)

56. Wang, Q. et al. Overexpression of a rice OsDREB1F gene increases salt, drought, and low temperature tolerance in both Arabidopsis and rice. Plant Mol. Biol. 67, 589-602 (2008)

57. Monfared, M. M. et al. Overlapping and antagonistic activities of BASIC PENTACYSTEINE genes affect a range of developmental processes in Arabidopsis. Plant J. 66, 1020-1031 (2011).

58. Di, L. L. et al. The SCARECROW gene regulates an asymmetric cell division that is essential for generating the radial organization of the Arabidopsis root. Cell 86, 423-433 (1996)

59. Vielba, J. M. et al. CSSCL 1 is differentially regulated upon maturation in chestnut microshoots and is specifically expressed in rooting-competent cells. Tree Physiol. 31, 1152-1160 (2011).

60. Nakamura, M. et al. Characterization of the class IV homeodomain-leucine zipper gene family in Arabidopsis. Plant Physiol. 141, 1363-1375 (2006).

61. Dicristina, M., Sessa, G. L., Linstead, P., Baima, S. \& Ruberti, I. The Arabidopsis Athb-10 (GLABRA2) is an HD-Zip protein required for regulation of root hair development. Plant J. 10, 393-402 (1996).

62. Söderman, E., Hjellström, M., Fahleson, J. \& Engström, P. The HD-Zip gene ATHB6 in Arabidopsis is expressed in developing leaves, roots and carpels and up-regulated by water deficit conditions. Plant Mol. Biol. 40, 1073-1083 (1999).

63. Olsson, A., Engström, P. \& Söderman, E. The homeobox genes ATHB12 and ATHB7encode potential regulators of growth in response to water deficit in Arabidopsis. Plant Mol. Biol. 55, 663-677 (2004).

64. Liu, W. et al. Genome-wide identification and expression profile of homeodomain-leucine zipper class I gene family in Cucumis sativus. Gene 531, 279-287 (2013).

65. Meister, A. \& Anderson, M. E. Glutathione. Annu. Rev. Biochem. 52, 711-760 (1983).

66. Salt, D. E. \& Rauser, W. E. MgATP-dependent transport of phytochelatins across the tonoplast of oat roots. Plant Physiol. 107, 1293-1301 (1995).

67. Li, H., Sun, Q., Zhao, S. \& Zhang, W. Principles and Techniques of Plant Physiological Biochemical Experiment. 1 edn, 119-120, 164-165, 167-169, 258-261 (Higher Education Press, 2000).

68. Young, M. D., Wakefield, M. J., Smyth, G. K. \& Oshlack, A. Gene ontology analysis for RNA-seq: accounting for selection bias. Genome Biol. 11, R14-R14 (2010).

69. Kanehisa, M. et al. KEGG for linking genomes to life and the environment Nucleic Acids Res. 36, D480-D484 (2008).

70. Mao, X., Cai, T., Olyarchuk, J. G. \& Wei, L. Automated genome annotation and pathway identification using the KEGG Orthology $(\mathrm{KO})$ as a controlled vocabulary. Bioinformatics 21, 3787-3793 (2005).

71. Chambers, M. C. et al. A cross-platform toolkit for mass spectrometry and proteomics. Nat. Biotechnol. 30, 918-920 (2012). 